\title{
PREPARAÇÃO E CARACTERIZAÇÃO DE CATALISADORES 10\% CO/MCM-41 SINTETIZADOS COM DIFERENTES FONTES DE SÍLICA
}

\author{
V. M. R. MENEZES ${ }^{1}$, L. A. LIMA $^{1}$ e M. G. F. RODRIGUES ${ }^{1}$ \\ ${ }^{1}$ Universidade Federal de Campina Grande, Departamento de Engenharia Química \\ E-mail para contato: virginia.any@gmail.com
}

\begin{abstract}
RESUMO - A Síntese de Fischer-Tropsch (SFT) na presença de um catalisador metálico suportado é atraente no setor industrial. O cobalto é um dos metais com atividade suficiente na SFT e maior resistência à desativação em relação a outros metais ativos. O objetivo deste trabalho é preparar catalisadores do tipo $10 \%$ Co/MCM-41 com diferentes fontes de sílica. A peneira molecular MCM-41 foi sintetizada com sílica aerosil 200 e argila chocolate B, com composição molar: 1,0 CTMABr:4,0 $\mathrm{SiO}_{2}: 1 \mathrm{Na}_{2} \mathrm{O}: 200 \mathrm{H}_{2} \mathrm{O}$. Os catalisadores suportados na MCM-41 foram obtidos via impregnação úmida com solução $0,1 \mathrm{M}$ de nitrato de cobalto, com volume adicionado relativo a $10 \%$ em peso do metal cobalto. As peneiras moleculares foram caracterizadas por Difração de Raios X (DRX), Adsorção física de Nitrogênio e Espectroscopia na região do infravermelho (IV). As análises de DRX indicaram que o cobalto foi encontrado na forma de $\mathrm{Co}_{3} \mathrm{O}_{4}$, mantendo a estrutura hexagonal da MCM-41 após incorporação do cobalto.
\end{abstract}

\section{INTRODUÇÃO}

Na última década o número de trabalhos de pesquisa que lidam com sílica mesoporosa tem crescido bastante (Berggren et al., 2005). O procedimento tradicional é utilizar um organosilicato simples como fonte de sílica, tais como o tetrametilortosilicato (TMOS) ou teraetilortosilicato (TEOS). No entanto, o processo tradicional apresenta a desvantagem de utilizar fontes de sílica caras e tóxicas. Recentemente, as considerações de ordem econômica e ambiental, despertou o interesse na utilização de silicatos inorgânicos baratos como material de partida. Os argilominerais com alto teor de $\mathrm{SiO}_{2}$ e $\mathrm{Al}_{2} \mathrm{O}_{3}$ podem ser considerados fontes alternativas para a síntese de peneiras moleculares, com um custo mais baixo e caracterizado como um processo verde (Yang et al., 2010).

Na preparação da MCM-41 são utilizados basicamente três precursores principais: um solvente, geralmente uma base; uma fonte de sílica, onde também podem ser utilizadas fontes alternativas de silício; e o agente direcionador da estrutura, o surfactante, que é um componente fundamental para a formação da estrutura característica (Schwanke et al., 2010). Este material 


\section{9 a 22 de outubro de 2014 \\ Florianópolis/SC}

possui área superficial específica acima de $700 \mathrm{~m}^{2} / \mathrm{g}$ e tamanho de poros de 2 a $10 \mathrm{~nm}$ (Beck et al., 1992).

A Síntese de Fischer-Tropsch (SFT) é uma reação química que pode ser utilizada para produzir hidrocarbonetos líquidos, principalmente combustíveis líquidos a partir do gás natural por conversão do gás de síntese (mistura de $\mathrm{CO}$ e $\mathrm{H}_{2}$ ) (Bessel, 1995; Yagi et al., 2005). Desde a década de 1990, o processo de FT tem demonstrado grande interesse por parte da indústria de petróleo, impulsionados pelo aumento das reservas de gás natural, pela procura mundial por combustíveis sintéticos e por legislações ambientais mais rígidas. O processo de Fischer-Tropsch baseia-se na conversão de gás de síntese em hidrocarbonetos sintéticos. Esta conversão é promovida por um reator contendo um catalisador metálico.

Os metais ativos típicos do processo são ferro e cobalto. O cobalto suportado é o catalisador preferido da SFT devido a sua alta atividade, seletividade a hidrocarbonetos pesados, baixa atividade de deslocamento de água e baixo custo. Além disso, a estrutura dos catalisadores de cobalto é controlada pelas propriedades químicas e textural do suporte, assim como, pelo método de preparação e interação do metal com o suporte (Dry, 2004).

O objetivo deste trabalho é a preparação e caracterização de catalisadores $10 \% \mathrm{Co} / \mathrm{MCM}-41$ com diferentes fontes de sílica, visando sua utilização na Síntese de Fischer-Tropsch.

\section{METODOLOGIA}

\subsection{Ativação Ácida da Argila Chocolate B}

A Argila Chocolate B utilizada na síntese da peneira molecular MCM-41 como fonte de sílica, proveniente da empresa BENTONISA - Bentonita do Nordeste S.A., situada no estado da Paraíba Brasil, foi passada em peneira de malha 200 mesh e submetida a um tratamento ácido: preparou-se uma solução de ácido sulfúrico 6M e adicionou-se a Argila chocolate B com razão de 1:6 sob agitação. $\mathrm{O}$ material foi mantido sob agitação à $100^{\circ} \mathrm{C}$ por 2 horas, com posterior secagem em estufa por 24 horas. $\mathrm{O}$ sólido final foi lavado com água deionizada e filtrado, atingindo um $\mathrm{pH}$ em torno de 7. O material foi seco em estufa por 24 horas.

\subsection{Síntese da Peneira Molecular MCM-41 Utilizando como Fontes de Sílica Argila Chocolate B e Sílica Aerosil 200}

A peneira molecular MCM-41 foi sintetizada pelo procedimento de tratamento hidrotérmico, baseada na patente de Melo et al. (2010), utilizando diferentes fontes de sílica: Sílica Aerosil e Argila Chocolate B. As peneira moleculares sintetizadas foram denominadas de acordo com cada tipo de fonte de sílica utilizada: MCM-41 SA (Sílica Aerosil) e MCM-41 ACB (Argila Chocolate B). A síntese foi conduzida preparando uma solução aquosa, contendo a fonte de sílica e de sódio (silicato de sódio), com agitação constante e temperatura compreendida entre 55 e $60^{\circ} \mathrm{C}$. A mistura resultante 


\section{9 a 22 de outubro de 2014 \\ Florianópolis/SC}

foi agitada até a completa homogeneidade por um período de 2 horas. Após este período, adicionou-se uma solução contendo o agente direcionador de estrutura brometo de cetiltrimetilamônio (CTMABr), a temperatura ambiente e sob agitação por um período de 1 hora.

O gel, de composição molar: 1,0 CTMABr:4,0 $\mathrm{SiO}_{2}: 1 \mathrm{Na}_{2} \mathrm{O}: 200 \mathrm{H}_{2} \mathrm{O}$, foi introduzido em um recipiente de teflon com tampa, inserido em uma autoclave de aço inox e aquecido a temperatura de $100^{\circ} \mathrm{C}$ em estufa, durante um período de 7 dias, controlando-se o pH do gel com ácido acético a cada 24 horas, mantendo-se na faixa de 9-10, até que o mesmo atingisse a estabilidade. O sólido final foi separado do sobrenadante por filtração, lavado com água deionizada e depois, seco em estufa.

\subsection{Preparação do Catalisador $10 \%$ Co/MCM-41}

O catalisador a base de Cobalto foi preparado a partir do processo de dispersão do metal nas peneiras moleculares MCM-41 (SA) e MCM-41 (ACB) por impregnação úmida, utilizando solução de $0,1 \mathrm{M}$ de nitrato de cobalto $\left(\mathrm{Co}\left(\mathrm{NO}_{3}\right)_{2} \cdot 6 \mathrm{H}_{2} \mathrm{O}\right)\left(\mathrm{Vetec}^{\circledR}\right)$. Para tal finalidade, adicionou-se a $5,0 \mathrm{~g}$ do suporte, o volume necessário da solução de $\left(\mathrm{Co}\left(\mathrm{NO}_{3}\right)_{2} \cdot 6 \mathrm{H}_{2} \mathrm{O}\right)$ relativo ao teor de $10 \%$ de metal empregado, sob agitação contínua a temperatura ambiente, por 30 minutos. O material obtido foi submetido a um processo de secagem em estufa a $80^{\circ} \mathrm{C}$ por um período de 24 horas. Após este período, o material foi calcinado, sob fluxo de nitrogênio com vazão de $100 \mathrm{~mL} / \mathrm{g}_{\text {cat }}$ min, sob uma rampa de aquecimento partindo da temperatura ambiente até $200^{\circ} \mathrm{C}$, com taxa de $10^{\circ} \mathrm{C} / \mathrm{min}$, permanecendo a $200^{\circ} \mathrm{C}$ por 1 hora. Neste período, o fluxo de nitrogênio foi trocado por ar sintético,

sendo a amostra aquecida a uma taxa de $2^{\circ} \mathrm{C} / \mathrm{min}$ de $200^{\circ} \mathrm{C}$ até $450^{\circ} \mathrm{C}$, permanecendo nesta última temperatura por 2 horas. Os catalisadores obtidos foram denominados de $10 \%$ Co/MCM-41 (SA) e $10 \% \mathrm{Co} / \mathrm{MCM}-41$ (ACB).

\subsection{Caracterização}

Difração de raios X (DRX): Os dados coletados foram das amostras utilizando o método do pó empregando-se um difratômetro Shimadzu XRD-6000 com radiação CuKa, tensão de $40 \mathrm{KV}$, corrente de $30 \mathrm{~mA}$, tamanho do passo de $0,0202 \theta$ e tempo por passo de $1,000 \mathrm{~s}$, com velocidade de varredura de $2^{\circ}(2 \theta) / \mathrm{min}$, com ângulo $2 \theta$ percorrido de $1,5^{\circ}$ a $10^{\circ}$ e 20 a $70^{\circ}$.

Adsorção física de nitrogênio (Método de BET): Na obtenção das propriedades texturais utilizou-se um aparelho ASAP 2020 da Micromeritics.

Espectroscopia na região do infravermelho (IR): A caracterização foi realizada usando um espectrômetro de infravermelho AVATAR TM 360 FT-IR E.S.P., com comprimento de ondas entre 4000 à $5000 \mathrm{~cm}^{-1}$ com resolução de $4 \mathrm{~cm}^{-1}$. 


\section{RESULTADOS E DISCUSSÃO}

Os espectros de absorção no infravermelho das amostras de MCM-41 (SA) e MCM-41 (ACB) estão apresentados na Figura 1.

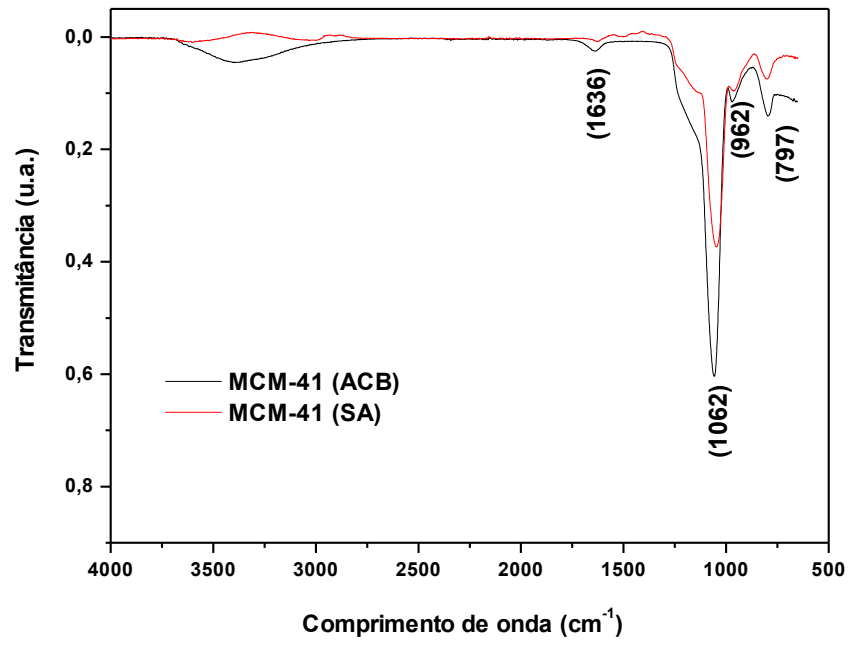

Figura 1 - Espectros de absorção no infravermelho das amostras: MCM-41 (SA) e MCM41 (ACB) calcinadas.

Os espectros de absorção no infravermelho das peneiras moleculares MCM-41 (SA) e MCM-41 (ACB) apresentam bandas na região de 500-1650 $\mathrm{cm}^{-1}$, características das vibrações fundamentais da rede da MCM-41 (Koh et al., 1997; Flanigen et al. 1971).

Na região de 1000 a $1250 \mathrm{~cm}^{-1}$ há uma banda larga referente ao estiramento assimétrico de Si-O-Si. Esta banda é composta de duas outras bandas, uma principal em $1062 \mathrm{~cm}^{-1}$, e outra banda em $1234 \mathrm{~cm}^{-1}$. A banda em torno de $797 \mathrm{~cm}^{-1}$ refere-se ao estiramento simétrico da ligação Si-O-Si na rede. Pelo espectro ainda pode ser observado uma banda de baixa intensidade a 962 $\mathrm{cm}^{-1}$. A MCM-41 apresenta esta banda devido ao elevado número de grupos silanóis em sua estrutura. De acordo com Clamblor e colaboradores (Camblor et al. 1993), esta banda deve ser atribuída ao estiramento da ligação $\mathrm{Si}-\mathrm{OH}$.

Os valores obtidos das propriedades texturais dos materiais sintetizados e a espessura da parede (e) são relatados na Tabela 1.

Tabela 1 - Propriedades texturais das amostras: MCM-41(SA) e MCM-41 (ACB) 


\begin{tabular}{|c|c|c|c|c|}
\hline Amostra & ${ }^{\mathbf{a}} \mathbf{S}_{\mathbf{B E T}}\left(\mathbf{m}^{\mathbf{2}} / \mathbf{g}\right)$ & ${ }^{\mathbf{b}} \mathbf{d}_{\mathbf{p}}(\mathbf{n m})$ & ${ }^{\mathbf{c}} \mathbf{V}_{\mathbf{p}}\left(\mathbf{c m}^{\mathbf{3}} / \mathbf{g}\right)$ & $\mathbf{e}(\mathbf{n m})$ \\
\hline MCM-41 (SA) & 863 & 3,6 & 1,05 & 1,7 \\
\hline MCM-41 (ACB) & 508 & 3,3 & 0,43 & 1,9 \\
\hline
\end{tabular}

${ }^{\mathrm{a}} \mathrm{S}^{\mathrm{BET} \text { : }}$ area calculada pelo método de BET; ${ }^{\mathrm{b}} \mathrm{d}_{\mathrm{p}}$ : Diâmetro de poro; ${ }^{\mathrm{c}} \mathrm{V}_{\mathrm{p}}$ : Volume total de poros; ${ }^{\mathrm{d}} \mathrm{e}$ : espessura da parede.

Observa-se elevada área superficial específica para amostra MCM-41(SA), no entanto a amostra MCM-41 (ABC) apresentou menor área superficial. Este fato pode ser explicado devido às impurezas remanescente do tratamento ácido da fonte de sílica (argila chocolate B). Em relação às dimensões dos poros, os dois materiais são semelhantes, e estes estão de acordo com os materiais sintetizados pelos pesquisadores da Mobil Oil Research and Development Co. (Kresge et al., 1992; Beck et al., 1992).

Os resultados de difração de raios $\mathrm{X}$ dos catalisadores Co/MCM-41 (SA) e Co/MCM-41 $(\mathrm{ABC})$ são apresentados nas Figuras 2 e 3 respectivamente.
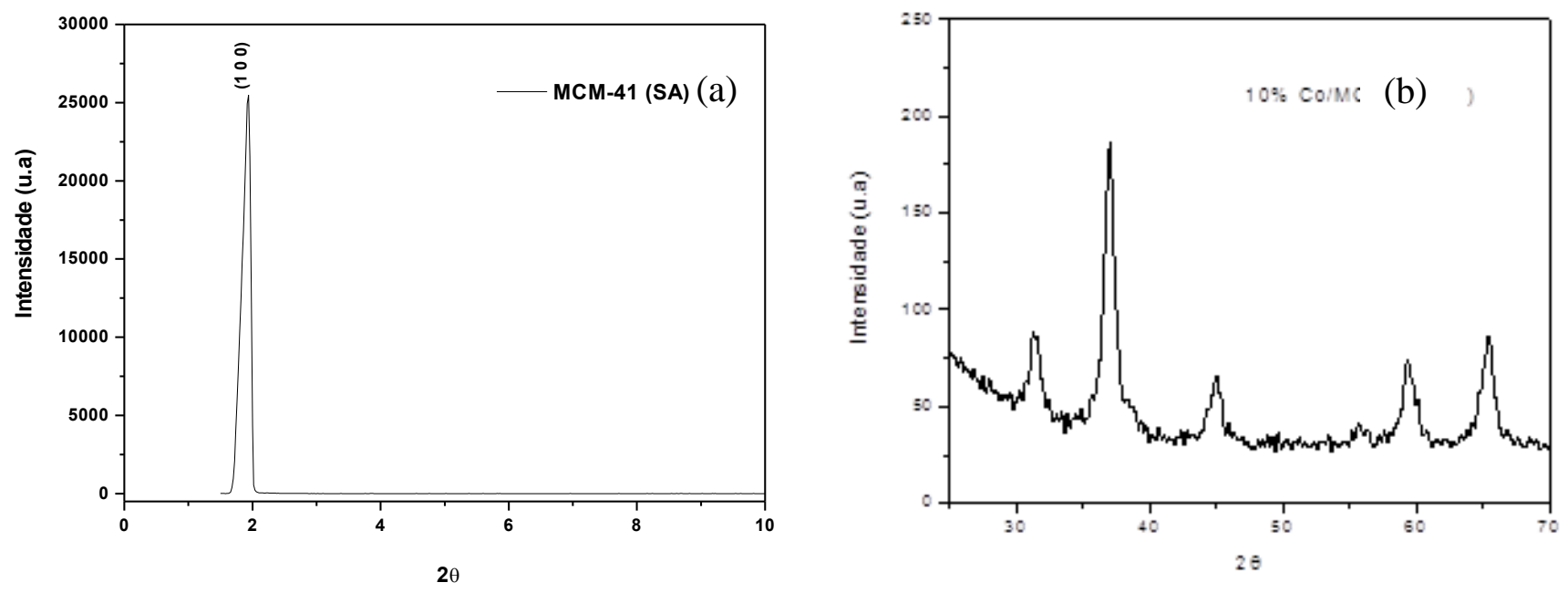

Figura 2 - Difratogramas de Raios X do catalisador 10 \% Co/MCM-41 (SA), após o processo de impregnação e calcinação (a) $2 \theta-1,5$ a $10^{\circ}$ (b) $2 \theta-20$ a $70^{\circ}$ 

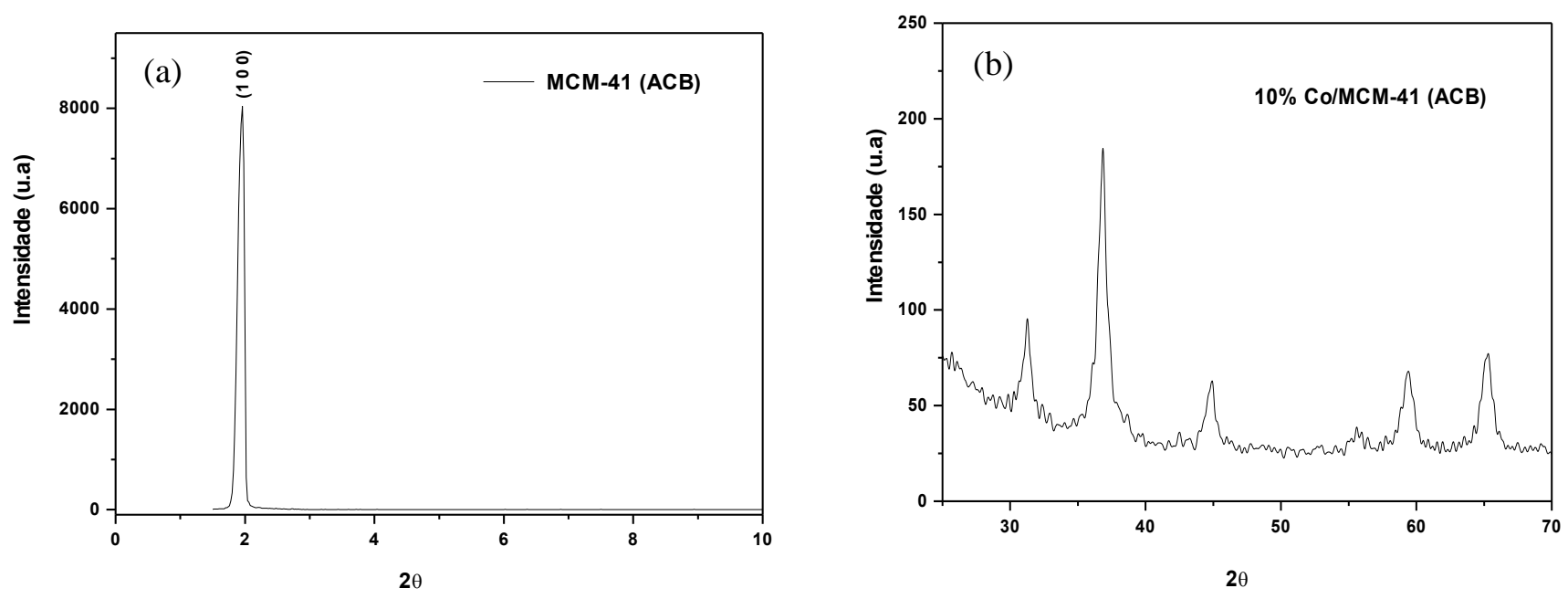

Figura 3 - Difratogramas de Raios X do catalisador $10 \%$ Co/MCM-41 (ACB), após o processo de impregnação e calcinação (a) $2 \theta-1,5$ a $10^{\circ}$ (b) $2 \theta-20$ a $70^{\circ}$

Para todas as amostras os difratogramas apresentados nas Figuras 2(a) e 3(a), apresentaram reflexões correspondentes ao plano de Miller (1 0 0) (Yang et al., 2010; Liou, 2011). Essa reflexão apresentada é devido aos tubos de sílica organizados na forma hexagonal formando um arranjo ordenado, indicando que após a impregnação e nova calcinação, não houve alteração da estrutura mesoporosa, mantendo assim o grau de ordenação. Diferentes intensidades foram encontradas nos difratogramas das amostras, apresentando o difratograma da MCM-41 (ACB) maior intensidade do que o difratograma da MCM-41 (SA).

Nas Figuras 2(b) e 3(b) são apresentados os picos entre $2 \theta=20^{\circ}$ e $70^{\circ}$, correspondentes ao óxido de cobalto formado da decomposição do sal (nitrato de cobalto) sobre as peneiras moleculares mesoporosas MCM-41(SA) e MCM-41(ACB), obtidos após o processo de calcinação das amostras impregnadas com o sal precursor $\mathrm{Co}\left(\mathrm{NO}_{3}\right)_{2} \cdot 6 \mathrm{H}_{2} \mathrm{O}$.

Os valores obtidos do espaço interplanar $d_{100}$ e o parâmetro de cela unitária $a_{0}$ dos catalisadores Co/MCM-41(SA) e Co/MCM-41(ACB) são apresentados na Tabela 2.

Tabela 2 - Espaço interplanar $\mathrm{d}_{100}$ e parâmetro de cela unitária $\mathrm{a}_{0}$ das amostras sintetizadas.

\begin{tabular}{|c|c|c|c|}
\hline Amostra & $\mathbf{2 \theta}(\mathbf{n m})$ & $\mathbf{d}_{\mathbf{1 0 0}}(\mathbf{n m})$ & $\mathbf{a}_{\mathbf{0}}(\mathbf{n m})$ \\
\hline Co/MCM-41 (SA) & $1,93^{\circ}$ & 4,7 & 5,3 \\
\hline Co/MCM-41 (ACB) & $1,96^{\circ}$ & 4,5 & 5,2 \\
\hline
\end{tabular}

Com base nos valores apresentados na Tabela 2 não foram observadas mudanças significativas nos parâmetros do arranjo hexagonal das amostras (MCM-41 (SA) e MCM-41 
(ACB)). Tais parâmetros estão de acordo com os encontrados na literatura (Melo et al., 2010; Stucky et al., 1997).

\section{CONCLUSÃO}

Os padrões de difração de raios X apresentaram picos característicos da estrutura MCM-41, evidenciando que após a impregnação com cobalto e calcinação, a estrutura da peneira molecular foi preservada, independentemente da fonte de sílica utilizada (SA ou ACB).

Pela análise textural das peneiras moleculares MCM-41 foram verificadas elevadas áreas superficiais específicas e dimensões de poro de acordo com a literatura, apresentando a peneira MCM-41 (SA) melhores resultados.

\section{AGRADECIMENTOS}

Os autores agradecem o suporte financeiro prestado pelas agências de fomento ANP/PRH-25 e a PETROBRAS pelo auxílio financeiro.

\section{REFERENCIAS}

BECK, J. S.; VARTUli, J. C.; ROTH, W. J.; LEONOWICZ, M. E.; KRESGE, C. T.; SCHMIDT, K. D.; CHU, C. T. W.; OLSON, D. H.; SHEPPARD, E. W.; MCCULLEN, S. B.; HIGGINS, J. B.; SCHLENKER, J. L., A new family of mesoporous molecular sieves prepared with liquid crystal templates. J. Am. Chem. Soc, v. 114, p. 10834-10843, 1992.

BERGGREN, A.; PALMQVIST, A. E. C.; HOLMBERG, K., Surfactant-templated mesostructured materials from inorganic silica. Soft Matter, v. 1, p. 219-226, 2005.

BESSEL, S. Investigation of bifunctional zeolite supported cobalt Fischer-Tropsch catalysts. Appl. Cat. A: Gen., v. 126, p. 235-244, 1995.

CAMBLOR, M. A.; CORMA, A.; PEREG-PARIENT, J., Infrared Spectroscopic Investigation of Titanium in Zeolites - A New Assignment of the $960 \mathrm{~cm}^{(-1)}$ Band. J. Chem. Soc., Chem. Commun., v. 6, p.557-559, 1993.

DRY, M. E. Present and future applications of the Fischer-Tropsch process. Appl. Catal. A: Gen., v. 276, p. 1-3, 2004.

FLANIGEN, E. M.; KHATAMI, H.; SZYMANSKI, H. A., Infrared structural studies of zeolite frameworks. Adv. Chem. Ser., v. 101, p. 201-207, 1971.

KOH, C. A.; NOONEY, R.; TAHIR, S., Characterisation and catalytic properties of MCM-41 
and Pd/MCM-41 materials. Catal Lett., v. 47, p. 199-205, 1997.

KRESGE, C. T.; LEONOWICZ, M. E.; ROTH, W. J.; VARTULIAND, J. C.; BECK, J. S., Ordered mesoporous molecular sieves synthesized by a liquid crystal template mechanism. Nature, v. 359, p. 710-715, 1992.

LIOU, T., A green route to preparation of MCM-41 silicas with well-ordered mesostructure controlled in acidic and alkaline environments. Chem Eng J., v. 171, p. 1458-1468, 2011.

MELO, D. M. A.; BARROS, J. M. F.; SOUSA, K. S. M. G.; MELO, M. A. F.; SILVA, M. L. P., Patent PI0901183-8 A2, 2010, 2.

SCHWANKE, A. J.; WITTEE, C.; BERTELlA, F.; PERGHER, S., Preparation and characterization of mesoporous materials Si-MCM-41 and Al-MCM-41. Persp. Erechim, v. 34, p. 99104, 2010.

STUCKY, G. D.; HUO, Q.; FIROUZI, A.; CHMELKA, B. F.; SCHACHT, S.; VOIGTMARTIN, I. G.; SCHÜTH, F., Directed synthesis of organic / inorganic composite structures. Stud. Surf. Sci. Catal., v. 105, p. 3 -28, 1997.

YAGI, F.; KANAI, R.; WAKAMATSU, S.; KAJIYAMA, R.; SUEHIRO, Y.; SHIMURA, M. Development of synthesis gas production catalyst and process. Catal. Today, v. 104, p. 2, 2005.

YANG, H.; DENG, Y.; DU, C.; JIN, S., Novel synthesis of ordered mesoporous materials AlMCM-41 from bentonite. Appl. Clay Sci, v. 47, p. 351-355, 2010. 